\title{
COMPARISON OF SINGLE- AND DOUBLE-SIDED PULSE WIDTH MODULATED SIGNALS WITH NON-LINEAR PREDISTORTION
}

\author{
Christopher Morrison ${ }^{1}$, Stephan Weiss ${ }^{2}$, Malcolm D. Macleod ${ }^{3}$ and Robert W. Stewart ${ }^{2}$ \\ ${ }^{1}$ Institute for System Level Integration, Edinburgh, Scotland, UK \\ 2 Dept of EEE, University of Strathclyde, Glasgow G1 1XW, Scotland, UK \\ ${ }^{3}$ QinetiQ Ltd, Greater Malvern, UK \\ christopher.morrison@sli-institute.ac.uk, \{stephan,bob\}@eee.strath.ac.uk
}

\begin{abstract}
Class D amplifiers are based on a low-bit pulse width modulated (PWM) signal reconstructed through the lowpass characteristic of the loudspeaker. To create a PWM signal, a non-linear conversion from a standard pulse code modulated $(P C M)$ signal has to be performed. The non-linear distortion created in this conversion process can be minimised by non-linear predistortion based on a Wiener-Hammerstein system. In this paper, we compare single and double-sided PWM conversion, whereby the former exhibits a higher resolution but also higher non-linearity compared to the latter. The trade-off between both approaches in creating a highly linear high performance class $D$ audio amplifier is demonstrated by means of simulations and an implementation.
\end{abstract}

\section{INTRODUCTION}

Digital class-D amplifiers use a pulse-width modulated signal to switch transistors, which represents a very efficient use of the power amplifier stage [1,2]. The demodulation of this this two-level signal is usually performed by the lowpass filter, which is implemented using passive components. The reduced cost and excellent efficiency of this approach have particularly triggered applications for the low-cost, lowperformance end of the market.

In order to apply class-D type amplifiers for the high-end market, non-linearities in the PWM conversion [3] need to be overcome, whereby two approaches have been proposed on the literature. Firstly, feedback correction of the amplifier output can be used to correct the PCM input $[4,5]$. Secondly, non-linear predistortion can be used to counteract nonlinear behaviour $[6,7,8,9,10]$. One particular predistortion technique is Wiener-Hammerstein systems which combine a simple polynomial non-linearity with linear filtering to create non-linear systems with memory [11]. Such WienerHammerstein systems have also been proposed in the context of audio amplifier predistortion [9, 12, 13], and will be the particular focus of this paper.

In this paper, we will follow a suggestion by [12, 13], where a Taylor series expansion of the distortion-inducing PWM conversion leads directly to a Wiener-Hammerstein model. Different models arise for single-sided and doublesided PWM. While single-sided PWM has a higher resolution than double-sided PWM, it introduces a higher level of

This work was performed as part of an Engineering Doctorate program, administered through the Institute for Systems Level Integration, at Linn Products Ltd., Glasgow, Scotland. The authors gratefully acknowledge the support of both ISLI and Linn Products Ltd. distortion and requires a stronger predistortion intervention. The aim of this paper is therefore to compare these two approaches, and compare theoretical and simulated results with measurements obtained from a system implementation. In terms of implementations, sufficiently fast digital signal processing devices such as TI's TMS320C6415 or the MityDSP board featuring a combination of a TMS320C6455 and an FPGA are explored.

The paper is organised as follows. In Sec. 2 the overall system setup is briefly outlined. The PCM-PWM conversion is described in Sec. 3, leading to the precoder reviewed in Sec. 4. We analyse simulations of the overall system in Sec. 5, comment on the implementation options, include measurements obtained from an implemented system. Conclusions are drawn in Sec. 6.

\section{OVERALL SYSTEM SET-UP AND MODEL}

The overall system set-up is the feedback-free architecture shown in Fig. 1. A PCM audio signal with 24 bits resolution sampled at $96 \mathrm{kHz}$ is passed through a linear-phase upsampler by a factor of 4 to a sampling rate of $f_{\mathrm{s}}=384 \mathrm{kHz}$. and a reduction to 8 bits word length. The noise-shaping block reduces the word length to 8 bits whilst preserving the noise floor in the audio range by way of sigma-delta techniques. Following the potential pre-distortion device, the 8 bit PCM signal is then passed through a PWM modulator, with a binary output with values \pm 1 operated at a sampling rate of $2^{8} \cdot 384 \mathrm{kHz}=98.304 \mathrm{MHz}$. This binary signal is power amplified fed to a loudspeaker, which acts as a lowpass filter.

The PCM/PWM conversion is a non-linear operation, which creates harmonic distortion. To compensate for the introduced distortion, a pre-distortion device is added to the system in Fig. 1. Note that the noise shaping block is transparent in the baseband, and operating the pre-distortion block first enables a resolution of 24 bits rather than 8 bits.

Even though the above system represents a multirate scenario, we assume for simplicity that all signals in Fig. 1 are represented at the same rate $f_{\mathrm{s}}$, and that the noise-shaping

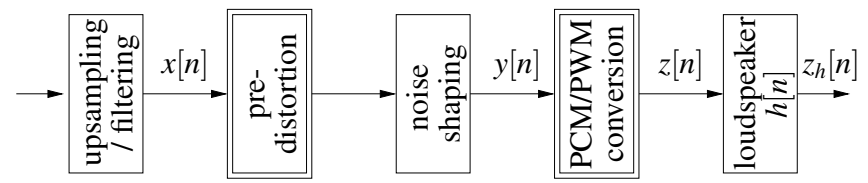

Figure 1: Overall system set-up. 


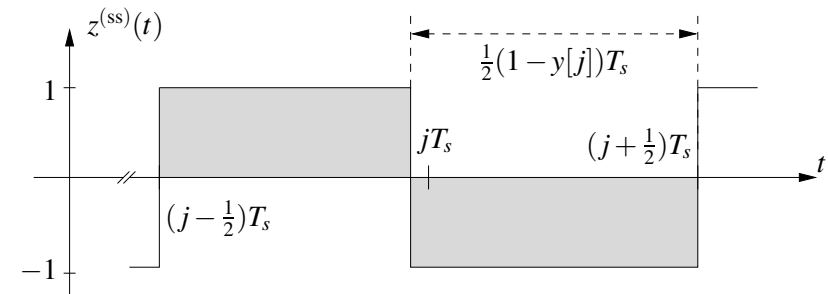

Figure 2: Output of a single-sided PWM conversion over one sampling period.

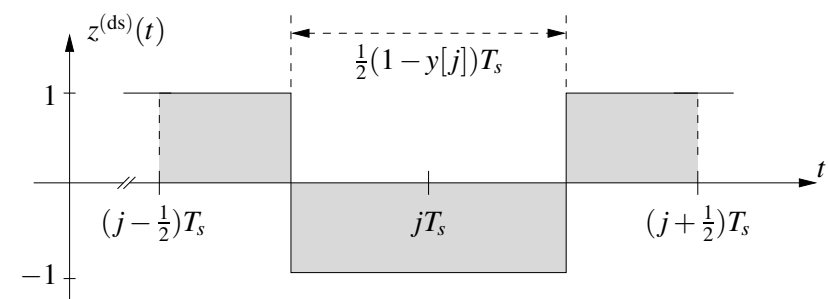

Figure 3: Output of a double-sided PWM conversion over one sampling period.

subsystem is transparent to the signal of interest. We further assume that the loudspeaker has a lowpass impulse response denoted by $h[n]$.

\section{PCM-PWM CONVERSION MODELS}

\subsection{Single-Sided PWM}

A simple 2-level universally sampled PWM digital modulator can be realised by comparing a pulse code modulation signal with a sawtooth running at the PCM sampling frequency $f_{s}$. The resolution of the sawtooth clock $f_{c}$ determines the precision of the conversion process.

Let $z(t) \in\{ \pm 1\}$ be the output of this single-sided PWM modulator, given an input $y[n]$. Firstly, we consider the convolution with a lowpass filter $h(t)$ at its output, $z_{h}^{(\mathrm{ss})}(t)=$ $z^{(\mathrm{ss})}(t) * h(t)$, and sample the signal at a sufficiently high rate $f_{\mathrm{s}}=T_{\mathrm{s}}^{-1}$ such that $z_{h}^{(\mathrm{ss})}[n]=z_{h}^{(\mathrm{ss})}\left(n T_{\mathrm{s}}\right)$. This single-sided PWM model for $z^{(\mathrm{ss})}(t)$ is shown in Fig. 2, and leads to the description

$$
z_{h}^{(\mathrm{ss})}[n]=\sum_{j=-\infty}^{\infty}\left\{\int_{-\frac{1}{2}}^{\frac{1}{2} y[j]} h[n-j-\tau] d \tau-\int_{\frac{1}{2} y[j]}^{\frac{1}{2}} h[n-j-\tau] d \tau\right\}
$$

for the sampled output. The PCM input is restricted to $|y[n]|<1$.

\subsection{Double-Sided PWM}

The model for a double-sided PCM-PWM conversion analogous to Sec. 3.1 is given in Fig. 3, which can be achieved by comparing the PCM signal with a triangular waveform. The output $z_{h}^{(\mathrm{ds})}[n]$ in this case consists of three segments,

$$
\begin{aligned}
z^{(\mathrm{ds})}[n]= & \sum_{j=-\infty}^{\infty}\left\{\int_{-\frac{1}{2}}^{-\frac{1}{4}+\frac{1}{4} y[j]} h[n-j-\tau] d \tau-\right. \\
& \left.-\int_{-\frac{1}{4}+\frac{1}{4} y[j]}^{\frac{1}{4}-\frac{1}{4} y[j]} h[n-j-\tau] d \tau+\int_{\frac{1}{4}-\frac{1}{4} y[j]}^{\frac{1}{2}} h[n-j-\tau] d \tau\right\}
\end{aligned}
$$

The drawback of this symmetric scheme is that the sampled PWM signal achieves a resolution reduced by one bit compared to the single-sided scheme on Fig. 2. However, this can be compensated through changes to the noise shaper.

\subsection{Taylor Series Expansion}

Consider PWM combined with the subsequent lowpass filter, based on (1) and (2). Performing a Taylor series expansion on the single-sided PWM according to Fig. 2 around the points $y[n]=0$ requires a lengthy derivation leading to the expression [14]

$$
z_{h}^{(\mathrm{ss})}[n] \approx \sum_{i=0}^{\infty} \frac{\left(-\frac{1}{2}\right)^{i}}{(i+1) !} h^{(i)}[n] * y[n]
$$

where $*$ is the convolution operator. The approximation in (3) holds in the low-frequency range, and $h^{(i)}[n]$ is the discretised version of the $i$ th differentiation of $h(t)$, i.e.

$$
h^{(i)}[n]=\left.\frac{\partial^{i} h(t)}{\partial t^{i}}\right|_{t=n T_{\mathrm{s}}} .
$$

The description in (3) is equivalent to the model derived for a trailing single-sided PWM in [13].

For a double-sided PWM conversion as shown in Fig. 3, the Taylor series expansion around $y[n]=0$ leads to [14]

$$
\begin{aligned}
z^{(\mathrm{ds})}[k] \approx & \sum_{v=1}^{\infty} \frac{1}{4^{2 v-2}(2 v-1) !} h^{(2 v-2)}[k] * x^{2 v-1}[k] \\
& -\frac{1}{4^{2 v}(2 v) !} h^{(2 \mu)}[k] * x^{2 v}[k] .
\end{aligned}
$$

The exact derivation [14] contains fractionally delayed derivatives $h^{(i)}[n]$, which can be approximated by the simplified expression (5) in the low-pass region. The double-sided system in [13], which uses an inverted model for Fig. 3 resulting in a different series expansion, can be shown to be equivalent.

\subsection{Wiener-Hammerstein Model}

Using the above Taylor series expansion, the PWM conversion system can be described by a parallel arrangement of Wiener-Hammerstein systems, whereby the differentiations (4) are expressed in terms of the lowpass filter $h[n]$ and $i$ th order differentiators $d^{(i)}[n], h^{(i)}[n]=d(i)[n] * h[n]$. In order to align differentiators in time, we assume the non-causal systems

$$
\begin{aligned}
& d^{(1)}[n]=\frac{1}{2} \delta[n+1] \quad-\frac{1}{2} \delta[n-1] \\
& d^{(2)}[n]=-\delta[n+1]+2 \delta[n]-\delta[n-1]
\end{aligned}
$$




$$
\begin{aligned}
z_{h}^{(S S)}[n] & =\left\{b_{0} y[n]+\frac{b_{1}}{2}\left(y^{2}[n+1]-y^{2}[n-1]+b_{2}\left(-y^{3}[n+1]+2 y^{3}[n]-y^{3}[n-1]\right)+\mathscr{O}\left(y^{4}\right)\right\} * h[n]=z^{(S S)}[n] * h[n]\right. \\
z^{(D S)}[n] & =b_{0} y[n]+b_{1}\left(-y^{2}[n+1]+2 y^{2}[n]-y^{2}[n-1]\right)+b_{2}\left(-y^{3}[n+1]+2 y^{3}[n]-y^{3}[n-1]\right)+\mathscr{O}\left(y^{4}\right)
\end{aligned}
$$

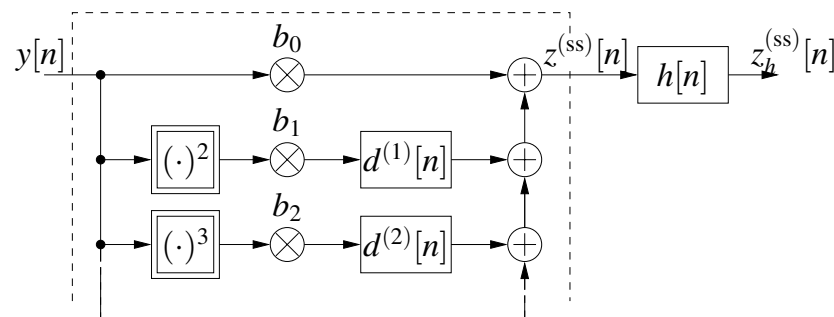

Figure 4: Hammerstein model of a single-sided PWM modulator (dashed box) including an $i$ th order differentiator $d^{(i)}[n]$, followed by the lowpass filter $h[n]$ which represents the loudspeaker.

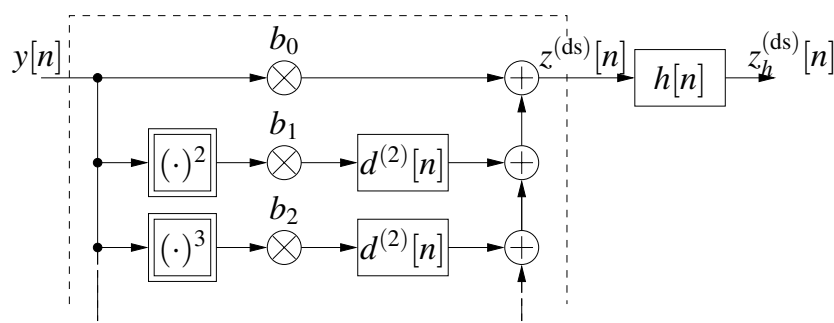

Figure 5: Hammerstein model of a double-sided PWM modulator (dashed box). Note that the 2 nd branch includes a 2 nd order derivative.

for the first two expansion terms. Note that (6) is a suitable approximation at low frequencies of a 1 st order derivative, which is justified due to subsequent filtering with $h[n]$. Note that the approximation of the first order derivative in (6) is correct at low frequencies. The non-causality is for notational convenience only, as the overall system can be made causal by introducing an appropriate overall delay.

This leads to a representation of the non-linear PWM conversion comprising of non-linear elements, differentiators of various orders, and a lowpass filter $h[n]$ as depicted in Figs. 4 and 5 for the single- and double-sided approaches. The overall outputs $z_{h}^{(S S)}[n]$ and $z_{h}^{(D S)}[n]$ given an input $y[n]$ are therefore characterised by (8) and (9), whereby the intermediate signal $z[n]$ denotes the input to the lowpass filter, $\mathscr{O}\left(y^{4}\right)$ refers to terms in $y[n]$ of 4 th or higher order.

\section{PREDISTORTION}

Following the approach in [13], a non-linear predistortion device approximating the inverse of the PWM expansion is selected to have the same structure as the models in Figs. 4 and 5 , whereby the infinite series in the PWM models are truncated to 3rd order. Assuming that $|x[n]|<1 \forall n$, we describe powers of $x[n]$ (or multiplicative combinations of differently delayed versions of $x[n]$ ) higher than cubic order by $\mathscr{O}\left(x^{4}\right)$, which are subsequently neglected.

\begin{tabular}{|r||c|c|c||c|c|c|}
\cline { 2 - 7 } \multicolumn{1}{c|}{} & \multicolumn{3}{c||}{ model } & \multicolumn{3}{c|}{ predistortion } \\
\hline mode & $b_{0}$ & $b_{1}$ & $b_{2}$ & $a_{0}$ & $a_{1}$ & $a_{2}$ \\
\hline \hline single sided & 1 & $-\frac{1}{4}$ & $\frac{1}{24}$ & 1 & $\frac{1}{4}$ & $-\frac{1}{24}$ \\
\hline double sided & 1 & $-\frac{1}{32}$ & $\frac{1}{96}$ & 1 & $\frac{1}{32}$ & $-\frac{29}{3072}$ \\
\hline
\end{tabular}

Table 1: Parameters of single-and double-sided PWM model and the coefficients of the predistortion filter.

\subsection{Single-Sided PWM Inversion}

For the single-sided case, inserting the predistortion output $y[n]=f(x[n])$ into (8) leads to (11), resulting in the selection

$$
\begin{array}{lll}
a_{0} b_{0} & =1 \\
a_{1} b_{0} & =-a_{0}^{2} b_{1} \\
a_{2} b_{0} & =-a_{0} b_{2}
\end{array} \quad \longrightarrow \quad \begin{aligned}
& a_{0}=b_{0}^{-1} \\
& a_{1}=b_{0}^{-3} b_{1} \\
& a_{2}=-b_{0}^{-2} b_{2}
\end{aligned}
$$

in order to create a unity gain for the linear terms and eliminate quadratic and cubic terms for the single-sided system. Cubic components with mixed lags cannot be cancelled but can be shown to exhibit a highpass characteristic, i.e. they will not interfer with the baseband signal of interest. Compared to the results in [13], (12) and (13) agree but (14) has a reversed sign due to a different approach compared to the single-sided PWM in Fig. 2.

\subsection{Double-Sided PWM Inversion}

For double-sided PWM, the insertion of a 3rd order nonlinear predistortion into (9) yields (15). Therefore, the selection

$$
\begin{array}{ll}
a_{0} b_{0}=1 & \longrightarrow a_{0}=b_{0}^{-1} \\
a_{1} b_{0}=-a_{0}^{2} b_{1} & \longrightarrow a_{1}=-b_{0}^{-3} b_{1} \\
a_{2} b_{0}=-a_{0}\left(a_{1} b_{1}+a_{0}^{2} b_{2}\right) \longrightarrow a_{2}=-b_{0}^{-1} a_{0}\left(a_{1} b_{1}+a_{0}^{2} b_{2}\right)
\end{array}
$$

results, whereby the uncancelled 3rd order term in line (15) is referred to as 3rd order residual. Based on the above sets of equations, coefficient sets for both single-and double-sided PWM are summarised in Tab. 1.

\section{SIMULATION, IMPLEMENTATION AND MEASUREMENTS}

This section reports on simulation results using different predistortion methods to linearise the PWM conversion, comments on the implementation and presents measurements obtained from an experimental class-D amplifier.

\subsection{System Simulations}

To test the system for different predistortion scenarios, uncorrelated, uniformly distributed noise with amplitude 


$$
\begin{aligned}
z^{(S S)[n]=} & b_{0}\left\{a_{0} x[n]+\frac{a_{1}}{2}\left(x^{2}[n+1]-x^{2}[n-1]\right)+a_{2}\left(-x^{3}[n+1]+2 x^{3}[n]-x^{3}[n-1]\right)\right\}+ \\
& +\frac{b_{1}}{2}\left\{a_{0}^{2} x^{2}[n+1]+\frac{a_{0} a_{1}}{2} x[n+1]\left(x^{2}[n+2]-x^{2}[n]\right)-a_{0}^{2} x^{2}[n-1]-\frac{a_{0} a_{1}}{2} x[n-1]\left(x^{2}[n]-x^{2}[n-2]\right)+\mathscr{O}\left(x^{4}\right)\right\} \\
& +b_{2}\left\{-a_{0} x^{3}[n+1]+2 a_{0} x^{3}[n]-a_{0} x^{3}[n-1]+\mathscr{O}\left(x^{4}\right)\right\} \\
= & a_{0} b_{0} x[n]+\frac{1}{2}\left(a_{1} b_{0}+a_{0}^{2} b_{1}\right)\left(x^{2}[n+1]-x^{2}[n-1]\right)+\left(a_{2} b_{0}+a_{0} b_{2}\right)\left(-x^{3}[n+1]+2 x^{3}[n]-x^{3}[n-1]\right) \\
& \quad+\frac{a_{0} a_{1} b_{1}}{4}\left\{x[n+1]\left(x^{2}[n+2]-x^{2}[n]\right)-x[n-1]\left(x^{2}[n]-x^{2}[n-2]\right)\right\}+\mathscr{O}\left(x^{4}\right)
\end{aligned}
$$

$$
\begin{aligned}
z^{(D S)}[n]= & a_{0} b_{0} x[n]+\left(a_{1} b_{0}+a_{0}^{2} b_{1}\right)\left\{-x^{2}[n+1]+2 x^{2}[n]-x^{2}[n-1]\right\} \\
& +2\left(a_{2} b_{0}+a_{0} a_{1} b_{1}+a_{0}^{3} b_{2}\right)\left\{-y^{3}[n+1]+2 y^{3}[n]-y^{3}[n-1]\right\} \\
& +\left(a_{0} a_{1} b_{1}\right)\left\{x[n+1]\left(x^{2}[n+2]+x^{2}[n]\right)-2 x[n]\left(x^{2}[n+1]+x^{2}[n-1]\right)+x[n-1]\left(x^{2}[n]+x^{2}[n-2]\right)\right\} \mathscr{O}\left(x^{4}\right)
\end{aligned}
$$

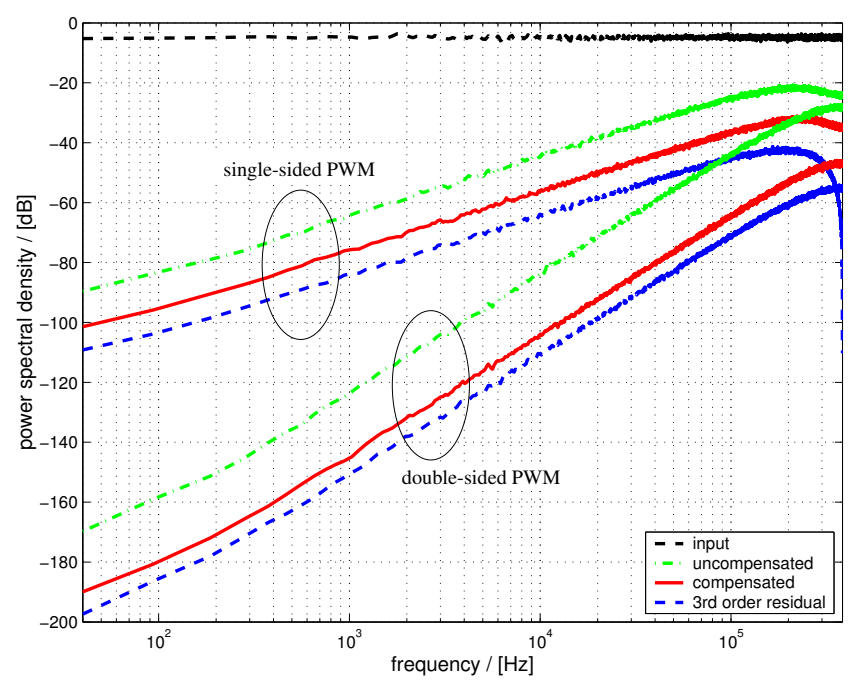

Figure 6: PSDs for system simulation with and without predistortion for both single- and double-sided PWM.

$|x[n]| \leq \frac{1}{2}$ is used as an excitation sequence. The constant power spectral densities (PSDs) in Fig. 6 indicate the input signal level for reference. In addition, PSDs for single- and double-sided PWM with and without non-linear predistortion are shown, whereby the 3rd order residuals of (11) and (15) indicate the maximum level of performance that can be expected with the truncation imposed on the predistortion devices. Overall, it is clear that the double-sided PWM exhibits a much enhanced performance.

For later reference, when excited by a $6.4 \mathrm{kHz}$ tone, the double-sided PWM system without and with predistortion yields the spectra shown in Fig. 7. The reduction of harmonic peaks at integer multiples of the excitation frequency is clearly visible.

\subsection{System Implementation}

A class-D implementation consists of the digital multirate system part shown in Fig. 1 and an H-bridge of transistors. One test board implementing the digital class-D power amplifier is shown in Fig. 8, with an FPGA performing the con-
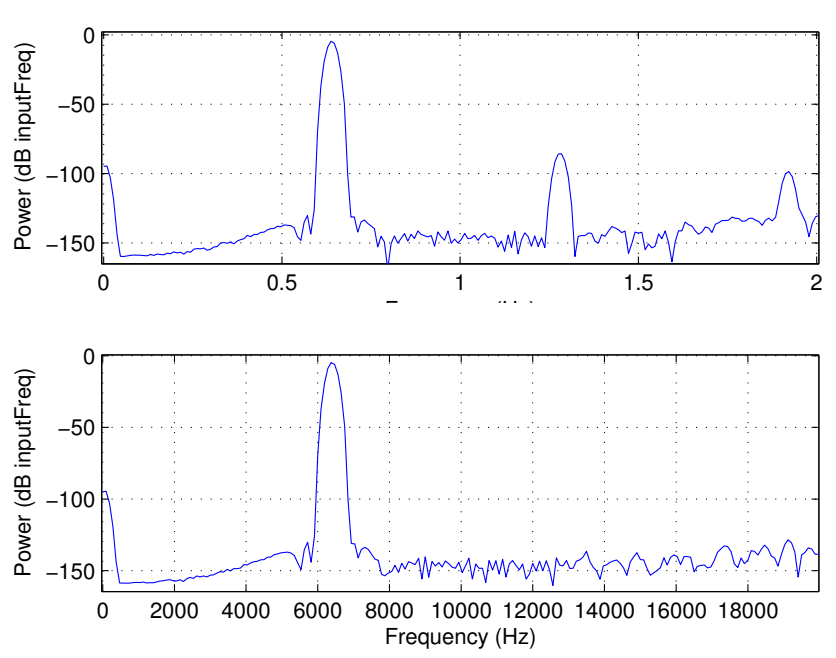

Figure 7: Digital power amplifier simulation using doublesided modulation (top) without and (bottom) with non-linear predistortion.

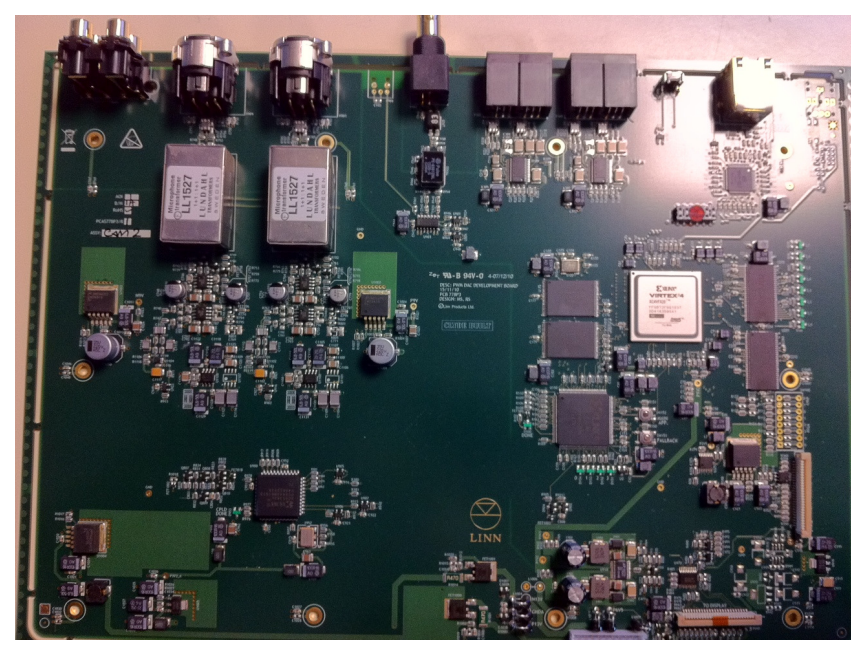

Figure 8: Digital class-D power amplifier test implementation, constructed for Linn Products Ltd., Glasgow, Scotland. 


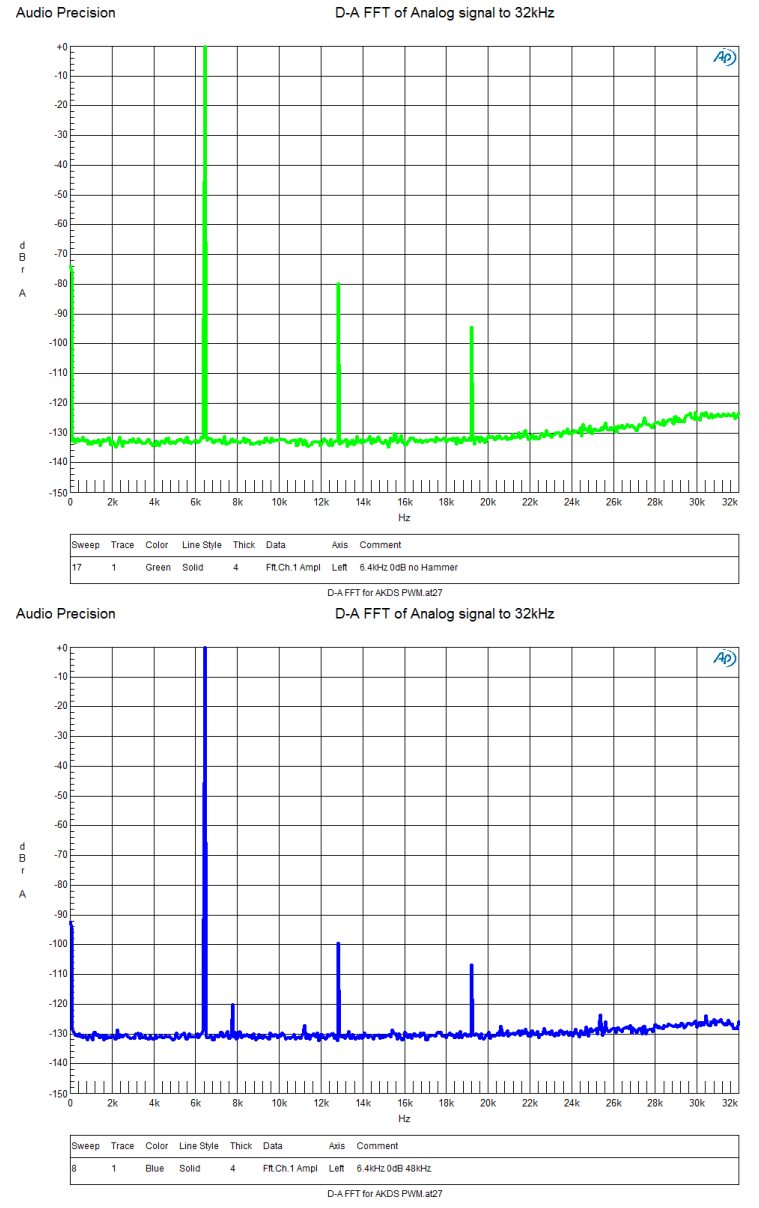

Figure 9: Spectrum analysis measurement of the class-D power amplifier output, based on the system, implementation in Fig. 8 using double-sided modulation (top) without and (bottom) with non-linear predistortion.

version processing, and a $100 \mathrm{MHz}$ output feeding into an $\mathrm{H}$-bridge and power amplifier stage. Typically, the processing requires significant filtering effort for the upconversion to $384 \mathrm{kHz}$, and powerful digital signal processing devices such as the Texas Instruments TMS320C4615 are a suitable option. A system currently under investigation is the MityDSP combining a high-performance 32-bit TMS320C6455 with an FPGA front-end for the PWM conversion operating close to $100 \mathrm{MHz}$.

\subsection{Measurements}

Spectrum analyser screen shots obtained from the test system operating in the absence and presence of a non-linear predistortion device for a double-sided PWM conversion are depicted in Fig. 9 for an input frequency of $6.4 \mathrm{kHz}$. The results indicate the harmonic structure resulting from non-linear distortion, and its reduction by approx. $20 \mathrm{~dB}$ when employing a 3 rd order non-linear Wiener-Hammerstein predistortion system. Note that this comes at the cost of a small raising of the general noise floor at low frequencies. Similar results, which are omitted to space limitation, can be obtained for the single-sided system [14], whereby harmonic levels are consistently higher.

\section{CONCLUSIONS}

Non-linear predistortion based on a Wiener-Hammerstein system obtained from a Taylor series expansion of singleand double-sided PWM conversion has been discussed. The linear dynamic range of a digital amplifier can be increased to satisfy the quality requirements placed on class-D amplifiers to be employed in high fidelity audio systems. Amongst the two modulation types, the double-sided approach, despite its one bit lower resolution, has been shown to achieve a significantly better performance in terms of linearity, particular when a non-linear predistortion device is utilised. Implementation options on a number of devices, including the TMS320C6415 or the TMS320C6455 in combination with FPGAs for the PWM conversion have been discussed.

\section{REFERENCES}

[1] M. B. Sandler. Digital-to-analogue conversion using pulse width modulation. Electronics \& Communication Engineering Journal, 5(6):339-348, June 1993.

[2] B. Putzeys. Digital audio's final frontier. IEEE Spectrum, 40(3):34-41, Mar. 2003.

[3] H. Mouton and B. Putzeys. Understanding the PWM nonlinearity: Single-sided modulation. IEEE Transactions on Power Electronics, 27(4):2116-2128, April 2012.

[4] P. Midya, T. Paulo, and B. Roeckner. High performance digital feedback for PWM digital audio amplifiers. In 121st Audio Engineering Society Convention, Oct. 2006.

[5] T. Mouton and B. Putzeys. Digital control of a PWM switching amplifier with global feedback. In 37th International Audio Engineering Society Conference, Aug. 2009.

[6] M. A. Gerzon. Predistortion techniques for complex but predictable transmission systems. J. Audio Eng. Soc, 20(6):475482, 1972.

[7] M. Sano and L. Sun. Identification of Hammerstein-Wiener system with application to compensation for nonlinear distortion. In 41st SICE Annual Conference, vol. 3, pp. 1521-1526, Aug. 2002.

[8] L. Ding, G. Zhou, D. Morgan, Z. Ma, J. Kenney, J. Kim, and C. Giardina. A robust digital baseband predistorter constructed using memory polynomials. IEEE Transactions on Communications, 52(1):159-165, Jan. 2004.

[9] P. Gilabert, G. Montoro, and E. Bertran. On the Wiener and Hammerstein models for power amplifier predistortion. In Asia-Pacific Microwave Conference, volume 2, page 4 pp., Dec. 2005.

[10] D. Morgan, Z. Ma, J. Kim, M. Zierdt, and J. Pastalan. A generalized memory polynomial model for digital predistortion of RF power amplifiers. IEEE Transactions on Signal Processing, 54(10):3852 -3860, Oct. 2006.

[11] K. Narendra and P. Gallman. An iterative method for the identification of nonlinear systems using a Hammerstein model. IEEE Transactions on Automatic Control, 11(3):546-550, July 1966.

[12] L. Risbo and H. K. Andersen. Conversion of a PCM signal into a UPWM signal. United States Patent US 6,657,566 B1, Dec. 2003.

[13] L. Risbo and H. K. Andersen. Conversion of a PCM into a UPWM signal. European Patent Specification EP0890221B1, July 2007.

[14] C. Morrison. Highly Linear High Performance Class D Amplifier Implementation. $\mathrm{PhD}$ thesis, Institute for System level Integration, 2012. 